\title{
Thiamine and Neuropsychiatry
}

\author{
Subir Bhattarcharjee \\ RMO-Cum-Clinical Tutor, Institute of Psychiatry, Kolkata
}

\section{INTRODUCTION}

Thiamine $\left(\underline{\mathrm{C}}_{12} \underline{\mathrm{H}}_{17} \mathrm{~N}_{4} \underline{\mathrm{OS}}\right)$ or vitamin $\mathrm{B} 1$, a sulfurcontaining vitamin, is a vitamin of the family ' $\mathrm{B}$ complex'. An essential nutrients, not synthesized within human body, exclusively has to replaced by dietary source to maintain optimal functioning of the central and peripheral nervous system as thiamine pyrophosphate (TPP) is an important coenzyme in the catabolism of sugars and amino acids. Subacute or chronic deficiencies due to many reasons were evidenced to cause various neuropsychiatric disorders from peripheral neuropathy, beriberi, Wernicke's encephalopathy, Korsakoff's syndrome to metabolic coma and death. As early deficiency conditions are reversible with thiamine replacement, the preventable and potentially treatable nature of these disorders makes this an important subject.

\section{CAUSES OF THIAMINE DEFICIENCY}

Thiamine deficiency can be caused by malnutrition, a diet high in thiaminase-rich foods (ferns, raw freshwater fish, shellfish) and / or foods high in antithiamine factors (tea, coffee, betel nuts). Chronic diseases, such as gastrointestinal diseases, persistent vomiting, alcoholism, Schizophrenia, depression, diabetes and chronic immune deficiency syndrome (AIDS), are often associated with grossly impaired nutritional status and so thiamine deficiency. [1] Dietary deficiencies may be due to dietary insufficiency, impaired intestinal absorption and possibly a genetically determined abnormality of transketolase. ${ }^{[2]}$

\section{DEFICIENCY SYNDROMES}

Role of vitamin deficiency, particularly vitamin B complex, in causation of various neuropsychiatric

conditions was suspected as early as during the description of pellagra like symptoms. Multiple vitamin deficiencies are often commonly found in psychiatric conditions. Among 172 successive admissions to a psychiatric unit, Carney ${ }^{[3]}$ found $30 \%$ to be deficient in thiamine, $27 \%$ in riboflavin and $9 \%$ in pyridoxine as assessed by red cell enzyme function assay and of them more than half were deficient in at least one of the three vitamin. Brozek and Caster ${ }^{[4]}$ in 1957 had done an experiment on the psychological effects of severe thiamine depletion. At first general weakness and extreme anorexia appeared which were associated with marked irritability. Depression was evidenced on scales of the MMPI and showed considerable deterioration. Further thiamine depletion ultimately led to peripheral neuropathy and impairment on tests of manual speed, coordination and reaction time. General intelligence was unaffected throughout the experiment. Thiamine replacement showed dramatic improvement in general characters though peripheral neuropathy improved more slowly.

\section{BERIBERI}

Chronic severe Thiamine (B1) deficiency classically leads to beriberi, with neuropathy, cardiac failure or peripheral edema, and neurological disturbances, fatigue, weakness and emotional disturbance, often appear much before the onset of physical symptoms. The three major forms of this disorder are dry beriberi, wet beriberi, infantile beriberi and a new form, gastrointestinal beriberi, was recognized recently. ${ }^{[1]}$

Dry beriberi is often characterized by calf muscle tingling, numbness to tenderness due to peripheral neuropathy in which there is symmetric impairment 
of sensory, motor, and reflex functions, affecting distal more than proximal limb segments. ${ }^{[5]}$ Peripheral neuropathy due to axonal neuropathy mimicking Guillain-Barré syndrome or large fiber proprioceptive central-peripheral axonal neuropathy presenting as a subacute sensory ataxia are also found in dry beriberi. ${ }^{[6]}$ In Wet beriberi peripheral neuropathy is often associated with symptoms of mental confusion, muscular atrophy, edema, tachycardia, cardiomegaly and congestive heart failure. ${ }^{[7]}$ Infantile beriberi occurs in infants, breast-fed by thiamin-deficient mothers who often do not show any thiamine deficiency signs. Infants show symptoms of cardiac disorder, most frequently tachycardia, aphonic, or pseudomeningitic forms of the disorder and sometime convulsions. ${ }^{[5]}$ Gastrointestinal beriberi is described of having symptoms like nausea, vomiting, abdominal pain, and lactic acidosis. ${ }^{[8]}$

Though rapid improvement occur following thiamine treatment, within 24 hours, peripheral neuropathy may require several months of thiamine treatment to improve. ${ }^{[9]}$

\section{WERNICKE'S ENCEPHALOPATHY (WE)}

This is a state of acute and severe thiamine deficiency and classically characterized by nystagmus, ataxia of gait, and a global confusional state, occurring together or in various combinations ${ }^{[10]}$ Wernicke first reported similar conditions in two cases of chronic alcoholism and in one with persistent vomiting after sulphuric acid poisoning. The age range is evenly distributed throughout adult life, with males affected approximately twice as often as females. Early thiamine therapy showed drastic improvement, particularly of the opthalmoplegias.

\section{ALCOHOLISM AND WERNICKE'S ENCEPHALOPATHY: CAUSES}

Chronic Alcoholism is one of the important causes of Wernicke's encephalopathy. In the developed world, WE is most commonly associated with alcohol misuse. Other than Alcoholism there are impaired absorption of thiamine from the gut, replacement of vitamin-containing foods by alcohol, impaired absorption of thiamine from the gut, impairment of storage by the liver, decreased phosphorylation to thiamine pyrophosphate (TPP), and excessive requirements for the metabolism of alcohol, known to precipitate WE There may be several other comorbid clinical conditions which may co-exist with alcohol use disorders, causing patients to be at additional risk of developing WE. (Table-1)

Lindboe and Løberg ${ }^{[17]}$ in 1998 showed that almost one-quarter of their postmortem cases were nonalcoholics, this rising to $40 \%$ in active acute cases. Most of the non-alcoholic patients had suffered from severe cachexia due to a variety of underlying diseases. But Victor ${ }^{[18]}$ found that Wernicke's encephalopathy as essentially a disease of alcoholics in American urban society as their largest series reported from the USA found that all but two of their 245 cases were suffering from established alcoholism. 
Table 1 : Additional risk of developing Wernicke's Encephalopathy ${ }^{[10]}$

- Teenage pregnancy with poor nutrition/drug misuse while mother still growing ${ }^{[11]}$

- Carbohydrate loading IV/oral when thiamine stores are minimal

- Diabetic ketoacidosis

- Chronic renal failure, dialysis, ${ }^{[12]}$

- Protein-calorie malnutrition from malabsorption

- Anorexia nervosa ${ }^{[13]}$

- Intravenous infusions including total parenteral nutrition without adequate thiamine

- Refeeding syndrome

- Thyrotoxicosis (very high thyroid hormone levels)

- Increased requirements caused by fever, pregnancy and adolescent growth

- Thiaminases are enzymes that break down thiamine in food (found in raw freshwater fish, raw shellfish, etc. - e.g. Japan)

- Genetic abnormality of transketolase enzyme

- Patients with protracted vomiting including pregnancy, toxemia

- AIDS, drug misuse

- Patients on diuretics for ascites

- Partial gastrectomy, gastrectomy or gastric stapling, gastric bypass, gastric or esophageal carcinoma, widespread carcinomas

- Severe obesity, ulcerative colitis, pernicious anemia

- Prisoners admitted to police cells, prison; individuals who are homeless or living in hostels

- Patients with Alzheimer's disease or neglect in old age, especially if living alone

- Chronic schizophrenia (paranoid) - self employed hunger strike ${ }^{[14,15]}$

- Widespread tuberculosis ${ }^{[16]}$

- Pernicious anemia ${ }^{[11]}$

Alcohol consumption does nothavelinear correlation with WE. Numerous factors play concurrently: the beverage consumed, its thiamine content, patterns of drinking and patterns of dietary neglect, personal susceptibility. Genetic vulnerability studies for WE have showed that transketolase is heterogeneous, existing as a number of isoenzyme variants and there are some difference in their affinity for TPP [19] There are also preliminary evidence for genetic transmission of reduced binding between TPP and transketolase in certain families. ${ }^{[2]}$ Clinical observation also proved that steady drinking extending over months or years and coupled with inadequate intake of food generally leads to WE and delirium tremens or other withdrawal syndromes had occurred at some time in the past in $40 \%$ of cases, withdrawal fits in $10 \%$ and liver disease in $10 \%$, indicating the general severity of alcohol abuse. ${ }^{[18]}$

\section{MAKING THE DIAGNOSIS}

Wernicke's encephalopathy is considered to be a underdiagnosed condition and may be present in the general population with a prevalence of around $2 \%$, and; probably, many cases do not have symptoms presumed to be associated with WE. ${ }^{[20]}$ Diagnosis of WE is dependent mainly on clinical observation. 
The classic triad of symptoms found in Wernicke's encephalopathy is :[21] 1) ophthalmoplegia (later expanded to other eye movement abnormalities, most commonly affecting the lateral rectus or any eye sign). 2) Ataxia (later expanded to imbalance or any cerebellar signs) 3) confusion (later expanded to other mental changes, having $82 \%$ incidence in diagnosis cases).

Caine established criteria that Wernicke's encephalopathy can be diagnosed in any patient with just two or more of the main symptoms. ${ }^{[22]}$ The sensitivity of the diagnosis by the classic triad was $23 \%$ but increased to $85 \%$ taking two or more of the four classic features. This criterion is not satisfactory as all the cases were studied in alcoholics.

In MRI-Brain reversible cytotoxic edema was considered to be the most characteristic lesion of WE though its sensitivity was found to be $53 \%$ and the specificity was $93 \%$. The location of the lesions was more frequently atypical among non-alcoholics, while typical contrast enhancement in the thalamus and the mammillary bodies was observed frequently associated with alcohol abuse. ${ }^{[23]}$ These abnormalities may include: Medial thalami, periaqueductal gray matter, mamillary bodies, and brainstem nuclei edema. Involvement is always found in bilateral symmetrical area. Value of DWI in the diagnosis of WE is minimal. Axial FLAIR MRI images represent the best diagnostic MRI sequence. Contrast material may highlight involvement of the mamillary bodies. CT scan brain was found to have very minimal role in diagnosis of WE. ${ }^{[24]}$

Thiamine can be measured using an erythrocyte transketolase activity assay or by activation by measurement of in vitro thiamine diphosphate levels. [24] Normal thiamine levels do not necessarily rule out the presence of $\mathrm{WE}$, as patient with difficulties in intracellular transport may show normal thiamine level.

\section{WHERE TO SUSPECT?}

Clinical evaluation of patients at risk of thiamine deficiency ${ }^{*[25]}$

\section{Clinical History}

- Weight loss in past year

- Reduced Body Mass Index

- General clinical impression of patient's nutritional status

- High dietary carbohydrate intake

- Recurrent episodes of vomiting in past month

- Co-occurrence of other nutritionally related conditions (polyneuropathy, amblyopia, pellagra, anemia)

\section{Early Signs-symptoms of Thiamine Deficiency}

- Loss of appetite

- Nausea/vomiting

- Fatigue, weakness, apathy

- Giddiness, diplopia

- Insomnia, anxiety, difficulty in concentration

- Memory loss 


\section{Later Signs-symptoms}

- Classic triad : oculomotor abnormalities, cerebellar dysfunction (ataxia) and confusion

- Quiet global confusion with disorientation in time/place

- Confabulation/hallucinations

- Onset of coma

*Patients may present with different combinations of symptoms and signs

\section{TREATMENT}

Most symptoms of WE generally improve quickly with early thiamine replacement therapy though Memory disorder may be permanent. ${ }^{[26]}$ As there is no known Thiamine toxicity, early intravenous or intramuscular thiamine therapy is always encouraging. Thiamine administration is usually continued until clinical improvement ceases. If glucose is given, such as in hypoglycemic alcoholics, thiamine must be given concurrently. If this is not done, the glucose will rapidly consume the remaining thiamine reserves, exacerbating this condition. ${ }^{[27]}$

Other supplements are also advised, including : cobalamin, ascorbic acid, folic acid, nicotinamide, zinc, phosphorus (dicalcium phosphate) as concurrent deficiencies is very common. ${ }^{[28]}$

\section{KORSAKOFF'S SYNDROME OR WERNICKE - KORSAKOFF SYNDROME (WKS)}

It is a manifestation of thiamine (vitamin B1) deficiency, generally due to toxic effect of alcohol, which is rather a spectrum of disorders which also encompasses beriberi, Wernicke's encephalopathy, and Korsakoff's psychosis and may manifest concurrently or separately. Only $20 \%$ of cases of WKS are identified before death and failure in diagnosis of WE and thus delayed or no treatment led to death in approximately $20 \%$ of cases, while $75 \%$ are left with permanent brain damage associated with WKS. ${ }^{[28]}$

WE and WKS bear the same risk and precipitating factors, including alcoholism, gastric carcinoma, intractable vomiting and severe dietary deficiency, which ultimately results in thiamine deficiency.

\section{CLINICAL FEATURE}

In WKS, there are Wernicke-encephalopathy (WE) phase, followed by the development of a chronic Korsakoff's syndrome(KS) phase. KS is characterized by acute onset of severe memory impairment without any dysfunction in intellectual abilities. ${ }^{[29]}$ The DSM IV lists the following criteria for the diagnosis of Korsakoff's Syndrome : 1.anterograde amnesia, 2. Variable presentation of retrograde amnesia and One of: Aphasia, Apraxia, Agnosia, A deficit in executive functions and there are, in addition, impairment of normal activities and function by the memory deficits and that the experience of amnesia must occur outside of times where the individual is in a state of delirium, intoxification, or withdrawal. The criteria for diagnosis also maintain that there must be evidence that the amnesia is caused by the use of alcohol. ${ }^{[29]}$

\section{COGNITIVE EFFECTS}

cognitive symptoms include insomnia, anxiety, difficulties in concentration, loss of memory for the immediate past, and gradual degeneration of mental state; consisting of confusion, confabulation, hallucinations and with increase in severity there are severely disrupted speech, giddiness and heavy headedness. ${ }^{[28]}$ In 2003 Brand, in their study on the cognitive effects of WKS, used a neuropsychological test battery which included tests of intelligence, 
speed of information processing, memory, executive function and cognitive estimation. They found that patients suffering from WKS showed impairments in all aspects of this test battery but most noticeably, on the cognitive estimation tasks. ${ }^{[30]}$

\section{MEMORY DEFICITS}

The amnesic symptoms of WKS include both retrograde and anterograde amnesia of recall or recognize information for recent events. WKS patients have also demonstrated difficulties in preservation as evidenced by a deficit in performance on the Wisconsin Card Sorting Test. ${ }^{[31]}$ Again it has been widely accepted that the anatomical structures that lead to the memory impairment in WKS are the mammillary bodies, and the thalamic regions. ${ }^{[32]}$

\section{CONFABULATION}

In WKS, spontaneous confabulation, incorrect memories that the patient holds to be true, and may act on, arising spontaneously without any provocation is seen more frequently than provoked confabulation. ${ }^{[33]}$ The spontaneous confabulations in WKS are thought to be produced by an impairment in source memory, where they are unable to remember the spatial and contextual information for an event, and thus may use irrelevant or old memory traces to fill in for the information that they cannot access.

\section{NEUROIMAGING FINDINGS}

Neuroimaging shows brain atrophy associated with WKS occurs in the regions of the mamillary bodies, the thalamus, the periaqueductal grey, and the walls of the 3 rd ventricle, the floor of the 4 th ventricle, the cerebellum, and the frontal lobe. Associated amnesia is generally due to atrophy in the structures of the diencephalon (the thalamus, hypothalamus and mamillary bodies), and is similar to amnesia that is presented as a result of other cases of damage to the medial temporal lobe. ${ }^{[34]}$

\section{TREATMENT}

Prompt administration of thiamine to patients with Wernicke's encephalopathy can prevent the disorder from developing into Wernicke-Korsakoff syndrome, or reduce its severity. Though Treatment will not completely reverse existing deficits, but can reduce the progression of the deficits caused by WKS. Patients suffering from WE should be given a minimum dose of $500 \mathrm{mg}$ of Thiamine hydrochloride, delivered by infusion over a 30 minute period for two to three days. If no response is seen then treatment should be discontinued but for those patients that do respond, treatment should be continued with a 250 $\mathrm{mg}$ dose delivered intravenously or intramuscularly for three to five days unless the patient stops improving. Such prompt administration of thiamine may be a life-saving measure. ${ }^{[27]}$

\section{REFERENCES}

Butterworth RF. Thiamin. In: Shils ME, Shike M, Ross AC, Caballero B, Cousins RJ, editors. Modern Nutrition in Health and Disease, 10th ed. Baltimore : Lippincott Williams \& Wilkins; 2006.

Mukherjee, A.B., Svoronos, S., Ghazanfari, A. et al. (1987) Transketolase abnormality in cultured fi broblasts from familial chronic alcoholic men and their male offspring. Journal of Clinical Investigation 79, 1039-1043.

Carney, M.W.P., Ravindrau, A., Rinsler, M.G. \& Williams, D.G. (1982) Thiamine, riboflavin and pyridoxine deficiency in psychiatric in-patients. British Journal of Psychiatry 141, 271-272.

Tanphaichitr V. Thiamin. In : Shils ME, Olsen JA, Shike M et al., editors. Modern Nutrition in Health and Disease. 9th ed. Baltimore: Lippincott Williams \& Wilkins; 1999.

Spinazzi M, Angelini C, Patrini C. Subacute sensory ataxia and optic neuropathy with thiamine deficiency. Nature Reviews Neurology. 2010; 6 :288-93

Mahan, L. K.; Escott-Stump, S., eds. (2000). Krause's food, nutrition, \& diet therapy (10th Ed.). Philadelphia : W.B. Saunders Company.

Maurice V, Adams RD, Collins GH. The Wernicke-Korsakoff Syndrome and Related Neurologic Disorders Due to Alcoholism and Malnutrition. 2nd ed. Philadelphia : FA Davis, 1989.

Duca, J., Lum, C., \& Lo, A. (2015). Elevated Lactate Secondary to Gastrointestinal Beriberi. J Gen Intern Med Journal of General Internal Medicine.

Victor, M., Adams, R.D. \& Collins, G.H. (1971) The WernickeKorsakoff Syndrome. Blackwell Scientific Publications, Oxford.

Cook CC, Hallwood PM, Thomson AD. B-vitamin deficiency and neuro-psychiatric syndromes in alcohol misuse. Alcohol Alcohol, $1998: 33$ : 317-336.

Spillane, J.D. (1947) Nutritional Disorders of the Nervous System. Livingstone, Edinburgh. 


\section{Thiamine and Neuropsychiatry}

Bergin, P.S. \& Harvey, P. (1992) Werncke's encephalopathy and central pontine myelinolysis associated with hyperemesis gravidarum. British Medical Journal 305, 517-518.

Ebels, E.J. (1978) How common is Wernicke-Korsakoff syndrome? Lancet ii, 781-782.

Pentland, B. \& Mawdsley, C. (1982) Wernicke's encephalopathy following hunger strike. Postgraduate Medical Journal 58, 427-428.

Spittle, B. \& Parker, J. (1993) Wernicke's encephalopathy complicating schizophrenia. Australia and New Zealand Journal of Psychiatry 27, 638-682.

Rimalovski, A.B. \& Aronson, S.M. (1966) Pathogenic observations in Wernicke-Korsakoff encephalopathy. Transactions of the American Neurological Association 91, 29-31.

Lindboe, C.F. \& Løberg, E.M. (1989) Wernicke's encephalopathy in non-alcoholics. An autopsy study. Journal of the Neurological Sciences 90, 125-129.

Greenwood, J., Jeyasingham, M., Pratt, O.E., Ryle, P.R., Shaw, G.K. \& Thomson, A.D. (1984) Heterogeneity of human erythrocyte transketolase : a preliminary report. Alcohol and Alcoholism 19, 123-129.

Isenberg-Grzeda E, Kutner HE, Nicolson SE (2012). “WernickeKorsakoff-syndrome : under-recognized and undertreated". Psychosomatics 53 (6) : 507-16.

Lough ME (June 2012). “Wernicke's encephalopathy: expanding the diagnostic toolbox". Neuropsychology Review 22 (2): 181-94

Caine, D; Halliday, G M; Kril, J J; Harper, C G (1 January 1997). "Operational criteria for the classification of chronic alcoholics: identification of Wernicke's encephalopathy". Journal of Neurology, Neurosurgery \& Psychiatry $62(1)$ : 51-60.

Zuccoli G, Pipitone N (February 2009). "Neuroimaging findings in acute Wernicke's encephalopathy : review of the literature". AJR. American Journal of Roentgenology $192(2): 501-8$.
Galvin R, Bråthen G, Ivashynka A, Hillbom M, Tanasescu R, Leone MA (December 2010). "EFNS guidelines for diagnosis, therapy and prevention of Wernicke encephalopathy". Eur. J. Neurol. 17 (12) : 1408-18

Carol Rees Parnish.(June 2009). Wernicke encephalopathy-Role of Thiamine. Practical Gastroenterology, Nutrition issues in Gastroenterology, Series \# 75 : 21-30

Goldman: Cecil Medicine, chapter 443, 23rd ed. 2007. Saunders, Elsevier.

Sechi G, Serra A (May 2007). “Wernicke's encephalopathy : new clinical settings and recent advances in diagnosis and management". Lancet Neurology 6 (5) : 442-55.

Kelley, Medicina Interna, page. 621, 974 ed.1990

Thomson, Allan D.; Marshall, E. Jane (2006). "The natural history and pathophysiology of Wernicke's Encephalopathy and Korsakoff's Psychosis". Alcohol and Alcoholism 41 (2) : $151-8$

Medline Plus Encyclopedia Wernicke-Korsakoff syndrome.

Brand, Matthias; Fujiwara, Esther; Kalbe, Elke; Steingass, Hans-Peter; Kessler, Josef; Markowitsch, Hans J. (2003). "Cognitive Estimation and Affective Judgments in Alcoholic Korsakoff Patients". Journal of Clinical and Experimental Neuropsychology 25 (3) : 324-34

Vetreno, Ryan Peter (2011). Thiamine deficiency-induced neurodegeneration and neurogenesis (PhD Thesis Binghampton University.

Zimitat, Craig; Nixon, Peter F. (1999). “Glucose loading precipitates acute encephalopathy in thiamin-deficient rats". Metabolic Brain Disease 14 (1) : 1-20.

Kessels, Roy P. C.; Kortrijk, Hans E.; Wester, Arie J.; Nys, Gudrun M. S. (2008). Confabulation behavior and false memories in Korsakoff's syndrome: Role of source memory and executive functioning. Psychiatry and Clinical Neurosciences 62 (2) : $220-5$.

Caulo, M. (2005). "Functional MRI study of diencephalic amnesia in Wernicke-Korsakoff syndrome". Brain 128 (7) : 1584-94. 